\title{
Portrait d'un fondateur : Julius Euting
}

Stuttgart 1839 - Strasbourg 1913

\section{Christophe Didier}

\section{CpenEdition}

Journals

Édition électronique

URL : https://journals.openedition.org/rbnu/3405

DOI : $10.4000 /$ rbnu.3405

ISSN : 2679-6104

Éditeur

Bibliothèque nationale et universitaire de Strasbourg

Édition imprimée

Date de publication : 1 novembre 2010

Pagination : 104-115

ISSN : 2109-2761

Référence électronique

Christophe Didier, «Portrait d'un fondateur : Julius Euting », La Revue de la BNU [En ligne], 2 | 2010, mis en ligne le 01 novembre 2010, consulté le 08 août 2021. URL : http://journals.openedition.org/rbnu/ 3405 ; DOI : https://doi.org/10.4000/rbnu.3405 Attribution - Pas d'Utilisation Commerciale - Partage dans les Mêmes Conditions 4.0 International. 


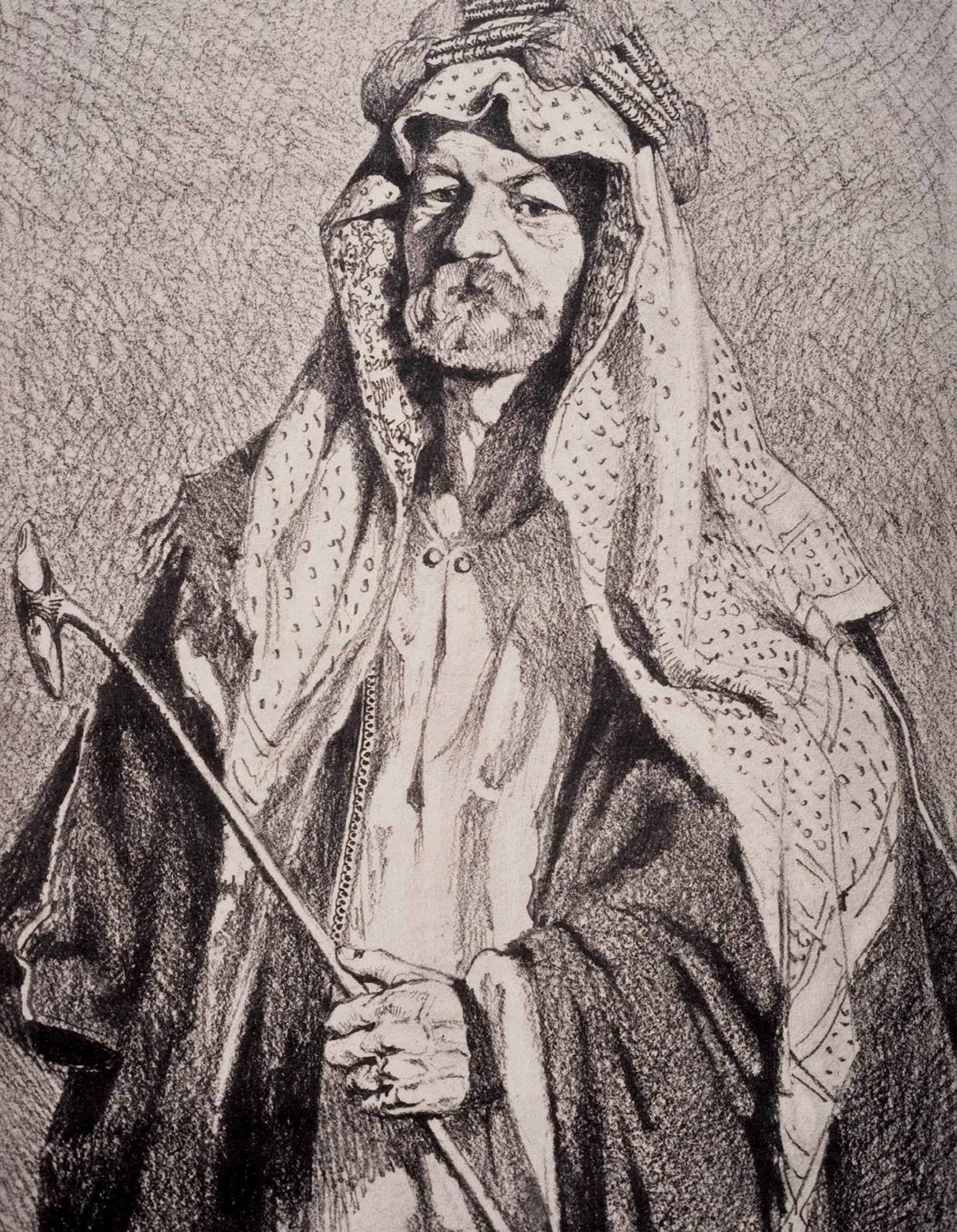




\section{Portrait d'un fondateur : Julius Euting}

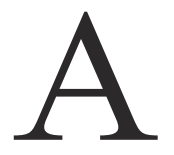

u début du siècle dernier, place Broglie à Strasbourg, deux hauts personnages frầchement arrivés en ville avisent un petit homme basané qui flâne apparemment sans but.

« Dites, mon brave, savez-vous où se trouve la rue ${ }^{* \star *}$ ?

- Mais oui, c'est justement ma direction.

Si ces messieurs veulent bien me suivre ".

Les étrangers rassurés suivent le petit homme.

"Puisque nous allons au même endroit, auriez-vous

l'obligeance de porter ma valise jusque-là ? " demande l'un des voyageurs.

« Mais bien sûr!

- Abuserai-je en vous demandant de porter aussi ma veste? " ajoute l'autre. "Il fait aujourd'hui une de ces chaleurs ! On se croirait en Afrique...

- En Afrique, certainement pas ", réplique le petit homme. « C'est encore pire là-bas.

- Encore pire! Mais comment pouvez-vous le savoir?

- Mais parce que j’y étais ".

Les deux personnages ne purent retenir un sourire en voyant à leurs côtés l'humble factotum.

" Tiens donc, vous y étiez ! Et à quelle occasion, si l'on peut savoir?

- Au cours d'une expédition. J’ai gratté quelques cailloux...

- Gratté des cailloux ? Et pourquoi donc?

- Mais pour pouvoir lire ce qu'il y a dessus ", répondit simplement le petit homme.

Ils étaient entre-temps arrivés au but. Ce dernier se défit alors de son fardeau, refusa énergiquement le pourboire qu'on lui tendait et non sans malice, salua les deux visiteurs par ces mots :

« Permettez-moi enfin de me présenter : professeur docteur Julius Euting, conseiller privé de Sa Majesté l'Empereur ". Il laissa là les deux visiteurs pantois, non sans leur avoir souhaité un agréable séjour à Strasbourg.

Quelques années auparavant, le même homme se trouvait, au cours d'un voyage dans la péninsule du Sinaï, l'hôte du célèbre monastère de SainteCatherine, sans doute à cette époque plus célèbre pour ses manuscrits anciens que par le confort de ses cellules. Prié par les moines de laisser quelques mots dans leur livre d'or, il leur demanda s'il pouvait les écrire dans sa langue natale, l'allemand. Les moines acceptèrent aussitôt, voyant là un honneur supplémentaire. L'étranger écrivit alors de sa plus belle plume : "J'aurais préféré mille fois cette nuit être l'hôte des chacals dans le désert plutôt que d'en être réduit à cet abominable nid à punaises ". L'histoire ne dit pas si les moines ont depuis appris l'allemand...

L'homme sur lequel ont pendant longtemps couru tant d'anecdotes (dont l'authenticité reste d'ailleurs à prouver) et qui autour de 1900 était devenu une petite célébrité dans sa ville d'adoption est aussi celui qui fit à l'époque de la BNU l'une des meilleures bibliothèques orientalistes d'Europe, l'enrichissant par exemple des fonds égyptiens qui sont à l'honneur dans ce numéro. Il fut au sens propre un des fondateurs de l'établissement qui, plus d'un siècle plus tard, accueille l'université d'été en papyrologie copte et s'enorgueillit d'avoir contribué à l'enrichissement du corpus de l'œuvre d'Empédocle. Faire son portrait aujourd'hui, c'est aussi retracer l'aventure qui vit naître un des plus beaux (et des plus originaux) fonds de bibliothèque français. 


\section{Une jeunesse souabe}

Julius Euting, bibliothécaire, directeur de 1900 à 1909 de la Kaiserliche Universitäts- und Landesbibliothek (l'ancêtre de la BNU), orientaliste et professeur honoraire de l'université de Strasbourg, mais aussi président pendant trente-trois ans du Club vosgien et membre fondateur de la Société de navigation aérienne de Strasbourg, naquit à Stuttgart en 1839. Il fut l'élève de l'Eberhard-Ludwig-Gymnasium de sa ville natale, puis du séminaire de Blaubeuren ${ }^{1}$ et enfin du célèbre Stift de Tübingen, le séminaire protestant qui avait accueilli avant lui des personnalités comme les poètes Hölderlin, Mörike, Uhland ou encore les philosophes Hegel et Schelling. Il y resta quatre ans, de 1857 à 1861 , et fut promu docteur le 20 février 1862 , avec un travail consistant en une traduction et un commentaire de la troisième sourate du Coran. C'est qu'en effet, dès la période de Blaubeuren, Euting s'était senti bien plus attiré par l'étude des langues anciennes que par celle de la théologie.

Cette tendance se confirma à

Tübingen, et le nouveau docteur n'eut de cesse, les années suivantes, de chercher à se perfectionner dans les études orientales. En 1863/64, il poursuivit sa formation à Paris, Londres et Oxford, tout en assurant sa subsistance en exerçant des fonctions de précepteur dans une famille noble du Wurtemberg.

\section{"Il vivait tout à fait à la Turque "}

Dès 1863 , il avait été nommé membre ordinaire de la Société allemande de l'Orient. Cependant, n'ayant pas à terme de moyens financiers suffisants pour poursuivre ses recherches sans travailler, il accepta la proposition qu'on lui fit en 1866 de devenir bibliothécaire et exerça son nouveau métier d'abord au séminaire protestant, puis à partir de juillet 1868 à la bibliothèque universitaire de Tübingen, qui était à l'époque hébergée dans le château de la ville. Euting $\mathrm{y}$ avait un logement de fonction consistant en deux pièces situées dans une des tours où déjà, si l'on en croit la chronique, il vivait à l'orientale au milieu des tapis, ne buvant jamais d'alcool mais fumant et buvant du café tant et plus. Il y recevait ses hôtes accroupi, la tête coiffée d'un fez rouge et s'adonnant aux plaisirs du narguilé.

En même temps, il poursuivait ses recherches et publia son premier ouvrage en $1867^{2}$. Cette annéelà fut aussi celle de son premier grand voyage vers l'Orient, voyage quelque peu improvisé et aventureux, puisqu'il conduisit Euting et deux Américains (dont l'un était alors étudiant en théologie à Tübingen) sur le Danube, à bord d'une embarcation à voile qu'ils avaient fait construire pour l'occasion - et dont ils revendirent le bois une fois arrivés à Budapest. De Donauwörth près d'Ulm, ils descendirent le fleuve, traversant l'Europe centrale, jusqu'à Constantinople et Smyrne, et revinrent, plus classiquement, par la Grèce et l'Italie après deux mois et demi d'absence.

\section{Sechzehsprochemännele}

Les grands voyages ont par la suite, à côté de son activité de bibliothécaire qu'il n'a jamais abandonnée, rythmé la vie d'Euting. En 1868, il visite la Norvège et la Suède ; en 1869, il est en Sicile et en Tunisie, où il effectue des recherches sur les antiquités carthaginoises ; en 1870, nouveau grand voyage en Sicile, à Athènes, Smyrne, Constantinople, Bucarest et la Transylvanie, Budapest et Vienne - la réplique inversée, en quelque sorte, de son expédition de 1867. Euting, qui était de très petite taille (il mesurait $1,50 \mathrm{~m}$.) mais que tous les témoignages de contemporains décrivent comme fort physiquement, avait incontestablement un caractère aventureux ; mais il est resté toute sa vie un savant actif, et le but premier de ses pérégrinations était scientifique. Comme spé- 
cialiste des langues orientales, c'était surtout l'épigraphie qui l'intéressait, et le relevé d'inscriptions puniques ou phéniciennes est l'objet de bon nombre de publications qui suivent ces premiers voyages. Surnommé plus tard à Strasbourg le "Sechszehprochemännele " (le petit homme aux seize langues), il s'était spécialisé dans les langues sémitiques, possédait parfaitement l'hébreu, le phénicien et l'araméen et parlait en outre couramment français, anglais et arabe - circonstance qui ne fut pas sans importance dans la conduite de ses voyages ultérieurs.

\section{Dans l'Alsace allemande}

A Tübingen, il s'était aussi occupé activement de bibliothéconomie ; le classement systématique de la bibliothèque universitaire fut ainsi établi par ses soins. C'était donc une personnalité qui commençait à être connue dans les milieux intellectuels du sudouest de l'Allemagne, lorsque la fin de la guerre de 1870 amena l'Alsace-Lorraine dans le giron du nouveau Reich. Le directeur de la nouvelle bibliothèque régionale et universitaire, Karl August Barack, forte personnalité lui aussi, recherchait sans aucun doute des collaborateurs à la hauteur des fonds qui s'étaient rapidement reconstitués après l'incendie de $1870^{3}$. Il fit appel à Euting pour en faire son premier bibliothécaire, et ce dernier prit ses fonctions le 16 juillet 1872 . Il ne devait quitter l'établissement que trente-huit ans plus tard, à sa retraite en 1909.

Il fut naturellement chargé du fonds orientaliste (publiant en 1877 le catalogue de la section arabe), mais fit aussi profiter la nouvelle bibliothèque des expériences acquises à Tübingen : le classement systématique est ainsi son œuvre, et profite aujourd'hui encore aux recherches dans le fonds ancien, puisque les livres sont restés physiquement classés dans l'état où ils étaient en 1918. En même temps, il s'intégra rapidement à la vie locale. En 1873, il est chargé de cours pour les langues sémitiques à l'université ; la même année, on lui demande d'enseigner l'hébreu aux élèves du Gymnase protestant, tâches d'enseignement qu'il conserva toute sa vie.

\section{Une vie à Strasbourg}

Le 9 novembre 1872, il fonde avec six autres personnes la section de Strasbourg du Club vosgien ${ }^{4}$, section qu'il dirigea de 1877 à 1887 . De 1876 à 1912 (avec une interruption de 1883 à 1886 due à ses voyages), il présida l'ensemble du Club. Trente-trois années de présidence, c'est aujourd'hui encore le plus long mandat que cette association ait connu. Euting était en effet très sportif, arpentant régulièrement les Vosges et la Forêt-Noire, dans des courses qu'apprécierait à leur juste valeur un bon randonneur d'aujourd'hui. Cette appétence à une vie sportive et de plein air (Euting était aussi bon patineur), couplée au fait qu'il ne boive jamais d'alcool lui font un mode de vie somme toute assez moderne pour son temps, où l'alcool faisait partie des codes presqu'obligatoires de la virilité et où les activités de plein air étaient encore peu répandues. Cela dit, l'activité d'Euting au sein du Club vosgien fut aussi scientifique : c'est à cette époque et sous son impulsion que furent réalisées les premières cartes de randonnées au $50.000^{\mathrm{e}}$, rendant accessible un massif jusque-là peu fréquenté. Euting, par ailleurs honnête peintre amateur, réalisa aussi de nombreuses illustrations destinées à des conférences (en particulier celles que donna August Schricker, le directeur du musée des arts décoratifs) et collabora au jadis célèbre guide des Vosges de Curt Mündel. Il fit paraître en outre une Description de la ville de Strasbourg et de la cathédrale (en 1881) qui connut seize rééditions, en français comme en allemand.

Euting n'était pas le plus mal placé pour parler des charmes du vieux Strasbourg. La bibliothèque, en attendant la construction d'un nouveau bâtiment (celui de la place de la République qui fut achevé en 1895) avait été hébergée au palais Rohan, où il bénéficiait d'un logement de fonction dans un pavillon de la partie nord-est du château. De fait, aujourd'hui encore, la vue sur le paysage urbain du palais, de ses terrasses et de la cathédrale en toile de fond, paysage entièrement artistique et minéral, est sans doute la plus belle de toute la ville. Dans ce logement de fonction aussi peu banal que celui qu'il avait occupé dans sa tour à Tübingen, Euting vivait dans son décor oriental, au milieu des objets rapportés de ses voyages et des 10 ooo livres de sa bibliothèque personnelle, profitant des conditions climatiques particulièrement favorables des terrasses pour y faire 
pousser toutes sortes de cactus. Il vécut là jusqu'à sa mort, une autorisation spéciale ayant été émise par Guillaume II pour qu'il puisse bénéficier de son logement même après sa mise à la retraite.

\section{Le grand voyage}

Ses publications épigraphiques ${ }^{5}$ régulières l'avaient fait connaître dans le monde savant, et il participait depuis 1874 aux congrès d'orientalistes qui se tinrent dans diverses capitales d'Europe et d'Afrique du Nord. Ses mérites scientifiques l'avaient fait nommer en 1880 professeur honoraire de la Faculté de philosophie de l'université de Strasbourg, titre qui du reste ne changea rien à son activité de bibliothécaire. Mais cette renommée naissante fut sans doute ce qui lui permit d'obtenir les moyens nécessaires au rêve qu'il caressait semble-t-il depuis longtemps : un voyage en Arabie.

L'occasion lui en avait été fournie par les récits d'un autre voyageur, le Français d'origine alsacienne Charles Huber. Celui-ci, lors d'une expédition entreprise quelques années plus tôt (en 1879/80), avait trouvé dans l'oasis de Teimâ des inscriptions sémitiques anciennes qu'il n'avait pu déchiffrer. Euting lui proposa alors d'y retourner avec lui, et obtint pour ce faire les moyens financiers nécessaires auprès du gouverneur d'Alsace-Lorraine et du roi du Wurtemberg. Il quitta Strasbourg le 22 mai 1883, et ne devait y revenir que quinze mois plus tard, le 16 août 1884 .

\section{Abd el-wahhâb}

C'est ce grand voyage, et la relation ${ }^{6}$ qu'il en publia quelques années plus tard, qui contribuèrent le plus à la renommée dont Euting bénéficia de son vivant. Arrivé à Damas en août 1883 , il se met alors en route à travers le désert, à dos de cheval puis de chameau, couchant le plus souvent à la belle étoile et se nourrissant de dattes, de riz, de pain local et de café. Pour combattre la chaleur et surtout pour ne pas attirer l'attention, Euting était habillé en Bédouin et se faisait appeler Abd el-wahhâb (serviteur du ToutPuissant). Sa petite taille, son teint hâlé et surtout sa parfaite connaissance de la langue arabe rendaient sans doute l'illusion assez probante. Les quelques portraits de cette époque que l'on a conservés de lui font de fait plus penser au membre d'un caravansérail qu'à un fonctionnaire de l'Empire allemand.

En se joignant le plus possible à des caravanes afin d'éviter les agressions, Euting et son guide atteignent leur premier but, l'oasis d'Hâjel (aujourd'hui Hajil en Arabie Saoudite) à $1000 \mathrm{kms}$ de Damas sur la route de La Mecque à Bagdad, où ils sont accueillis par l'émir Ibn Raschîd chez qui ils restent trois mois. Les raisons de cette longue halte, inutile du strict point de vue épigraphique (les sites où Euting voulait travailler se trouvaient encore à $450 \mathrm{kms}$ d'Hâjel) sont obscures, de même que restent obscurs les motifs de Huber : parti au départ sans Euting, il le rejoignit pour s'en séparer peu après. Huber voulait-il précéder son compagnon dans la découverte des inscriptions ? Quoi qu'il en soit, il n'eut pas de chance dans son entreprise et mourut au début de 1884 lors d'une attaque de brigands.

Euting atteint quant à lui son but, les inscriptions sur les falaises et les grottes de Teimâ, el Hegr et el-Öla. Afin d'en rapporter des estampages lui permettant de les déchiffrer une fois de retour à Strasbourg, il s'était fait fabriquer un matériel ad hoc, une échelle démontable de huit mètres de long et de longs rouleaux de papier fabriqués pour la circonstance. Ce papier abondamment mouillé était ensuite appliqué à l'aide d'une brosse sur la face à reproduire. La brosse le faisant pénétrer dans toutes les anfractuosités de la pierre reproduisait ainsi en relief l'inscription, qu'on retrouvait après avoir laissé sécher, puis retiré la feuille. Euting a longuement décrit (et illustré) ces séances d'estampage sous le vent et le soleil impitoyables - et aussi sous les regards de badauds importuns, plus fréquents qu'on ne l'imagine dans ces contrées désertiques...

Lors de son voyage, il eut à affronter de nombreuses tentatives d'attaques de Bédouins. La plus sérieuse eut lieu sur le chemin du retour en direction de la mer Rouge, où un groupe d'une dizaine de brigands l'obligea à se servir de ses armes. Il tua deux adversaires au cours du combat, ce qui lui fit dire par la suite qu'il était sans aucun doute le seul ancien séminariste de Tübingen qui ait deux morts par balles sur la conscience. En tous les cas, cette circonstance lui fit hâter son retour et, après huit mois et 2300 kms de chevauchée en Arabie, il regagna Strasbourg depuis l'Egypte. 


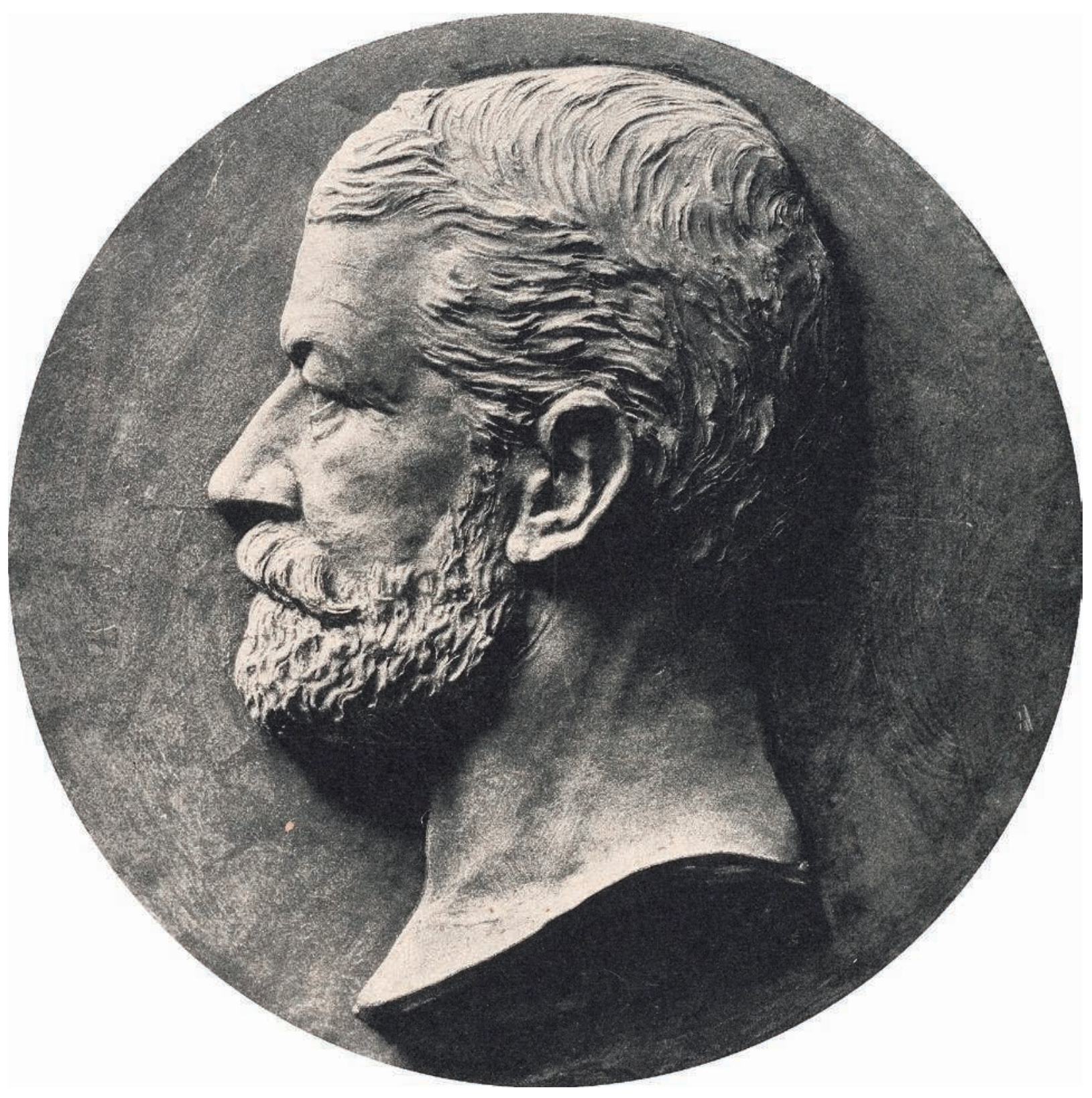

Médaillon de bronze (autour de 1890) représentant Euting et dessiné par le sculpteur et médailliste Walther Eberbach (1866-1944) (coll. BNU) 


\section{A Guillaume II qui lui demandait comment il avait pu voyager dans le désert d'Arabie pendant des semaines sans pouvoir se laver, l'eau étant sévèrement comptée, Euting répondit : "Majesté, quand l'homme a le choix entre se transformer en squelette ou en cochon, il choisit généralement la seconde solution "}

\section{Butin épigraphique}

A son retour l'attendent accueil officiel, conférences, articles de presse... Le bibliothécaire est devenu une petite célébrité. Mais le butin scientifique de ce voyage ne doit pas être minimisé : Euting rapporte en effet 900 estampages d'inscriptions araméennes, nabatéennes, sabéennes, lihyanites (cette dernière écriture $^{7}$ ayant en grande partie dû sa redécouverte à son zèle), dont l'étude va l'occuper toute sa vie, à côté de ses fonctions de bibliothécaire. Les premiers résultats de ses recherches paraissent d'ailleurs peu après, en 1885 , sous le titre Inscriptions nabatéennes d'Arabie. Les mérites d'Euting dans les domaines de l'épigraphie et de la paléographie des pays du Proche et Moyen-Orient, et particulièrement dans l'étude des langues et écritures sémitiques, semblent avoir été réels si l'on en croit les témoignages d'orientalistes de renom, dont plusieurs furent ses collègues ou élèves, comme les professeurs Littmann ou Seybold de Tübingen. C'est donc en parfait connaisseur de ces domaines et de leurs pays d'origine qu'il constituait à la bibliothèque des collections qui en firent bientôt un établissement de référence, héritage qui lui profite encore aujourd'hui.

Il refit par la suite d'autres voyages : en 1889 et 1890 , il part à deux reprises pendant plusieurs mois en Egypte, au Sinaï et en Syrie du Nord ; en 1898, il accompagne l'expédition Brünnow lors d'un voyage de trois mois au Proche-Orient, voyage au cours duquel il découvre vraisemblablement les ruines de la Mschatta qui allait donner lieu par la suite à une petite polémique strasbourgo-berlinoise ; en 1903, il passe à nouveau deux mois en Palestine. Au cours de tous ces voyages, il tient des journaux dont seul celui de l'expédition de $1883 / 84$ a été publié. Les autres sont aujourd'hui conservés sous leur forme manuscrite à la bibliothèque universitaire de Tübingen. De 1869 à 1905, Euting a ainsi tenu 26 journaux et 9 carnets d'esquisses.

\section{La Mschatta}

Le 9 mai 1902, Guillaume II en visite à Strasbourg se rend à la bibliothèque, où il est accueilli par Euting qui en était le directeur depuis 1900. Au cours de la conversation, ce dernier montre au souverain des photographies prises par Brünnow d'un monument étonnant, un palais omeyade construit au milieu du VIII ${ }^{e}$ siècle et situé à une quarantaine de kilomètres au nord-est de la mer Morte. Il suggère d'en faire faire des moulages pour le nouveau Kaiser-FriedrichMuseum que l'on construit alors à Berlin ; ce à quoi Guillaume II rétorque qu'il préfère se faire offrir l'original par le sultan ottoman Abdul Hamid II, qui régnait alors sur cette région. Oubli ou négligence de sa part, il ne dit par contre pas à Euting que ces photographies lui ont déjà été montrées quelques mois plus tôt par Wilhelm von Bode, le directeur des musées royaux de Berlin, et que des études ont été diligentées par ce dernier pour évaluer la possibilité d'un transport de la ruine à Berlin ${ }^{8}$. Quoi qu'il en soit, les négociations s'accélèrent après l'entrevue strasbourgeoise, et le démontage (retardé par le choléra qui sévit alors en Syrie et en Palestine) commence en septembre 1903. Euting fait à cette occasion un de ses derniers grands voyages et arrive sur le site de la Mschatta le jour où partent les dernières caisses, le 5 octobre 1903. Le transport du monument s'effectua en effet dans 422 caisses, qui transitèrent par Damas, Beyrouth et Hambourg, avant d'arriver à Berlin le 24 décembre 1903. La Mschatta est aujourd'hui une des pièces les plus célèbres de la section islamique du Pergamonmuseum de Berlin. 


\section{Polémiques}

L'arrivée du monument ne passe pas inaperçue dans le monde de l'art et jusqu'au grand public. Dans le Schwäbischer Merkur du 7 avril 1904 paraît un article où Euting raconte sa rencontre avec Guillaume II et laisse ainsi entendre qu'il a eu dans l'affaire un rôle déterminant. Après le dévoilement de la Mschatta au public en octobre de la même année, deux articles du Strassburger Post reprennent cette thèse et insistent sur le rôle d'Euting dans l'acquisition du monument. Cette publicité agace à Berlin, et Bode y répond, également dans le Strassburger Post, dans un article intitulé "Suum cuique!" (à chacun son dû) où il tente de remettre les choses au point.

On admet aujourd'hui qu'au moment où Euting présentait les photos à Guillaume II, la décision de ramener la Mschatta était déjà prise et les négociations avec le sultan entreprises. Mais il n'empêche que c'est bien après la visite strasbourgeoise du 9 mai 1902 que les ministères concernés ont véritablement mis en branle le processus et que les premières autorisations furent délivrées par le gouvernement ottoman. Le rôle déclencheur joué par Euting, que Guillaume II appréciait beaucoup, ne doit donc pas être sous-estimé et sa place dans l'acquisition d'une pièce maîtresse des musées de Berlin, si elle n'est pas la première, doit malgré tout être évaluée comme il se doit.

\section{Fin de carrière}

En 1900, après la mort de Barack, il devient à 61 ans et après 30 ans comme bibliothécaire à Strasbourg, directeur de la Kaiserliche Universitäts- und Landesbibliothek. Il conserva ce poste neuf ans, jusqu'à sa mise à la retraite. Son activité de directeur a donné parfois lieu à des débats, certains lui reprochant d'être trop peu administratif et en particulier trop peu rigoureux face à certains dépassements budgétaires. On a même parlé à l'époque de " système Euting " pour décrire l'épreuve de force qu'il engageait ainsi avec sa tutelle. Mais après sa mort, on lui reconnaissait ce mérite d'avoir montré ce faisant qu'une grande bibliothèque représente d'abord un enjeu financier. Dans ce sens, sa gestion un peu particulière a sans doute préparé utilement le terrain de son successeur? ${ }^{9}$.
On a aussi reproché à Euting d'avoir excessivement favorisé le secteur oriental et de n'avoir pas été assez encyclopédique dans ses vues. Jusqu'où ces reproches sont justifiés, il apparaît aujourd'hui difficile de le dire, la dimension encyclopédique de la BNU étant un fait plutôt bien établi. Quoi qu'il en soit, Euting prit comme directeur deux décisions importantes dans l'histoire des collections : il la fit entrer en 1902 dans le cartel des papyrus (voir à ce sujet les articles du dossier) et acquit en 1905 une partie importante de l'héritage d'Arthur de Gobineau, faisant de la BNU aujourd'hui encore l'établissement de référence pour qui s'intéresse à son œuvre et à sa pensée ${ }^{10}$.

Avec l'âge était venu le temps des honneurs : depuis 1898, il était membre correspondant de l'Institut de France et de l'Académie des inscriptions et belleslettres ; en 1907, il fut nommé membre de l'Académie royale prussienne des sciences de Berlin. En 1909, pour son $70^{\mathrm{e}}$ anniversaire, deux cents amis et connaissances se cotisèrent pour lui offrir un portrait en relief qui fut apposé sur la tour du Climont (entre les vals de Villé et de Schirmeck dans les Vosges), appelée aujourd'hui encore "Juliusturm " en son honneur. Mais quoique notable par certains aspects, il garda toujours une certaine touche d'originalité qui fit qu'on le trouvait parfois là où on ne l'aurait pas forcément attendu : il fit ainsi partie des membres fondateurs de la Société de navigation aérienne de Strasbourg et entreprit en novembre 1898, à presque soixante ans, son premier grand voyage en ballon qui le mena jusqu'à $3400 \mathrm{~m}$. d'altitude de Strasbourg à Colmar. Il réédita l'expérience dix ans plus tard, en mars 1909, en survolant la Forêt-Noire jusqu'à Rottenburg, au bord du Neckar où le ballon atterrit dans un champ de houblon.

A sa retraite qu'il prit à 70 ans, il continua ses travaux épigraphiques, mais s'occupa surtout à mettre en ordre le deuxième tome de son récit de voyage en Arabie (qui parut de façon posthume en 1914).

Affaibli par une maladie respiratoire peut-être due à sa forte consommation tabagique (il avait réduit celle-ci dans les dernières années à... 14 cigares par jour), il mourut le 2 janvier 1913.

\section{Un enterrement en montagne}

Le dimanche $1^{\text {er }}$ juin 1913, sur les pentes du Seekopf, sommet de la Forêt-Noire du Nord alors peu fréquenté 


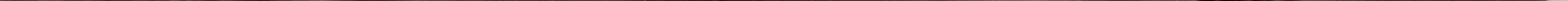


des touristes, se pressait une foule inhabituelle venue rendre un dernier hommage à celui qui avait décidé, depuis longtemps déjà, de se faire enterrer au milieu des forêts. Euting, qui fréquentait volontiers ces lieux, avait sollicité - et obtenu - du roi du Wurtemberg la possibilité de disposer d'un lopin de terre pour y installer sa sépulture. Le souverain lui avait octroyé dès 1903 un are à cet effet, et l'enclos et la pierre tombale avaient été aménagés dès 1905. Euting avait aussi depuis longtemps choisi son inscription funéraire et l'avait fait graver sur une plaque de marbre blanc qu'il conservait dans sa chambre à coucher ; traduite par ses soins de l'arabe, elle disait : " Il est le Vivant, l'Eternel. Quand mon lit sera réduit en poussière et que je séjournerai auprès du Seigneur miséricordieux, alors félicitez-moi, mes amis, et dites : 'bonne nouvelle pour toi, tu es arrivé auprès d'un Etre de bonté '". Cette plaque n'alla cependant pas à l'endroit qui lui était destiné, son propriétaire craignant au bout du compte qu'elle ne soit dégradée ou recouverte d'inscriptions par des touristes peu scrupuleux. Il la légua pour finir au musée Linden de Stuttgart, où elle fut malheureusement détruite au cours des bombardements de la Seconde Guerre mondiale.

\section{L'héritage}

Dans son testament, établi longtemps avant sa mort, Euting léguait à la bibliothèque ses estampages d'inscriptions hébraïques, des moulages de monnaies et de gemmes, des lettres adressées à lui, ainsi que des objets antiques de plus grand format (pierres portant des inscriptions, autels et une momie). Sa bibliothèque personnelle ne présentait pas d'intérêt pour l'institution, qui possédait déjà ces ouvrages : elle fut vendue à la firme Fock de Leipzig. En revanche, la KULB acquit sa collection de monnaies et de poids de verre. Quant aux objets orientaux, ainsi que son portrait en Bédouin par Léon Hornecker, ils allèrent au musée Linden de Stuttgart, où ils subirent le même sort que la pierre tombale au cours des bombardements. Enfin, il légua à un musée du Wurtemberg ${ }^{11}$, pour remercier le roi qui lui avait octroyé l'emplacement pour sa pierre tombale, la plus grande part de ses estampages orientaux.

Afin de laisser de lui un souvenir, il avait également souhaité instituer une fondation originale : chaque personne qui viendrait se recueillir sur sa tombe le jour de son anniversaire aurait droit, en récompense de ses efforts (la tombe est à $1041 \mathrm{~m}$. d'altitude...), à une tasse de café à l'auberge du Ruhestein. La Première Guerre mondiale et l'inflation qui suivit eurent vite raison du petit capital de 500 marks laissé par Euting, et la " Kaffeestiftung " ne fonctionna réellement qu'une fois, le 11 juillet 1913. Il avait aussi institué un prix de 400 marks récompensant chaque année l'élève du Eberhard-Ludwig-Gymnasium de Stuttgart qui se serait le plus distingué dans l'art calligraphique. Cette deuxième fondation ne survécut pas plus que la première au conflit mondial, mais fut réanimée en 1960 par le fils d'un de ses amis (et l'un de ses biographes allemands) sous le nom d'Euting-Graner-Stiftung.

\section{Sic transit gloria mundi}

L'isolement voulu par Euting pour sa dernière demeure lui valut, comme on pouvait s'y attendre, quelques vicissitudes post mortem. Ainsi, un journal de la Forêt-Noire notait en 1953 que les détritus divers laissés par les touristes en avaient bien souillé les abords. En 1980, le Club vosgien fait état de la volonté du Schwarzwaldverein ${ }^{12}$ de remédier à l'état d'abandon du lieu et d'installer une plaque commémorative pour renseigner les promeneurs. " La tombe contenant les cendres d'un inconnu [...] a longtemps échappé aux regards ", lit-on dans Les Vosges, qui profite de la circonstance pour rappeler brièvement qui était Euting - preuve que son nom n'éveillait plus guère de souvenirs. De fait, la tombe fut entièrement restaurée en 1980 , et l'on remit à cette occasion la plaque contenant l'inscription arabe - telle qu'on peut la voir encore aujourd'hui.

A la bibliothèque devenue BNU, la mémoire d'Euting ne s'est jamais vraiment perdue, mais son héritage a dû parfois patienter avant d'être réellement exploité : ainsi les estampages hébraïques, réalisées par Euting à l'occasion des découvertes qu'il avait faites lors des travaux d'agrandissement de Strasbourg, n'ont été redécouverts qu'en 1981 par le professeur Schwartz, alors custode du cabinet numismatique de la BNU.

Personnalité incontestablement originale, sans doute le seul bibliothécaire qui ait laissé son nom sur deux sommets de montagne, Julius Euting fait partie de ces quelques noms qui ont contribué à la vitalité culturelle de Strasbourg entre 1870 et 1918, à 
l'égal des Georg Dehio, Georg Simmel, Wilhelm von Bode, Anton Seder ou Adolf Michaelis. La BNU lui doit l'organisation ancienne de ses collections, socle sur lequel elle fonctionne toujours aujourd'hui, et le caractère oriental d'une partie de son fonds ancien, qui en fait un établissement original dans le paysage de l'enseignement supérieur français. En ce sens, il a bien été un fondateur, dont l'héritage continue aujourd'hui de stimuler ses descendants - ce numéro de La Revue de la BNU en est le plus bel exemple.

\section{Christophe Didier}

\section{Notes}

1 - Petite ville du Jura souabe, à $80 \mathrm{kms}$ environ de Stuttgart, célèbre aujourd'hui encore pour son monastère médiéval et les magnifiques retables qu'il abrite.

2 - Qolasta ou Chants et leçons sur le baptême et la migration de l'âme, Stuttgart, 1867

3- Après l'incendie de l'ancienne bibliothèque dû au bombardement de la ville en 1870 , le nouveau pouvoir allemand avait rapidement souhaité fonder un nouvel établissement, et avait, outre un appel à la générosité internationale, solidement doté celui-ci, de sorte que sa collection était déjà imposante quelques années après le sinistre initial.

4- Le Club vosgien a été fondé en octobre 1872 par Richard Stieve, un Allemand qui avait été nommé juge au tribunal de Saverne un an plus tôt, et créa la section de Saverne.

5 - Le catalogue sur fiches de ses écrits, articles et contributions divers, qu'il avait dressé chez lui et entretenait soigneusement, comprenait à la fin de sa vie 202 titres.

6 - Cette relation (Tagebuch einer Reise in Inner-Arabien) fut publiée en deux temps : le premier volume parut en 1896, le second (à titre posthume) en 1914 .

7 - L'écriture lihyanite, alphabet nord-arabique de transition entre le phénicien ancien et le sabéen, fut utilisée environ 1000 ans avant Mahomet. Elle était quasiment inconnue avant qu'Euting n'en fasse un des objets de ses études.

8 - On craignait en effet que la Mschatta ne serve de carrière pour la construction d'une ligne de chemin de fer en cours dans la région. Ces craintes des archéologues n'étaient pas totalement infondées, un castel romain situé non loin de là ayant subi pareil sort un peu auparavant

9 - C'est en tout cas l'analyse qu'on trouve dans le portrait d'Euting tracé à l'occasion de sa mort dans la revue professionnelle Zentralblatt für Bibliothekswesen. C'est aussi ce que sous-entend Dubled dans son Histoire de la Bibliothèque nationale et universitaire de Strasbourg (voir bibliographie).

10 - Sur le fonds Gobineau, voir par exemple le catalogue de l'exposition Impressions d'Europe (Strasbourg, 2003).

11 - L'information, trouvée dans un recueil d'articles (sans indication de leurs provenances !) ne précise pas lequel. On peut penser qu'il s'agit du musée Linden de Stuttgart, auquel cas ces estampages ont sans doute subi le même sort que les autres objets d'Euting conservés dans ce musée.

12 - L'équivalent du Club vosgien pour la Forêt-Noire, fondé en 1864.

\section{ORIENTATIONS BIBLIOGRAPHIQUES}

Allocution sur la mort d'Euting, in Académie des inscriptions et belles-lettres. Comptes rendus des séances de l'année 1913, séance du 17 janvier 1913

An Julius Eutings Grabstätte, in Aus dem Schwarzwald, $\mathrm{n}^{\circ} 7$, juillet 1913

Dem Andenken des Ruhesteinvaters Julius Euting, in Fremdenblatt für Freudenstadt und Umgebung, $\mathrm{n}^{\circ}$ 4, 22 juin 1913

DUBLED Henri / Histoire de la Bibliothèque nationale et universitaire de Strasbourg. Strasbourg, 1973

ENDERLEIN Volkmar / Die Erwerbung der Fassade von Mschatta, in Staatliche Museen zu Berlin - Preussischer Kulturbesitz, Forschungen und Berichte, Band 26, 1987

Erinnerungen an Julius Euting, in Deutsche Tageszeitung, $\mathrm{n}^{\circ}$ 6, 4 janvier 1913

GRANER Hans / Julius Euting : Bibliothekar, Forschungsreisender und Orientalist, in Lebensbilder aus Schwaben und Franken, 8. Band (Stuttgart : Kohlhammer, 1962)

HUCK Joseph-Louis / Julius Euting, in Les Vosges et le Club vosgien : autour d'un centenaire, 1872-1972. Strasbourg : Club vosgien, 1972

HUMMEL Herbert / Geist und Kirche : Blaubeurer Klosterschüler und Seminaristen. - [S.l.] : Alb-Donau-Kreis, 1998

NOTZ Hermann / Sechzehnsprachenmännle, Ruhesteinvater und Feuerteufel : Professor Dr. phil. Julius Euting. - Freudenstadt :

Schwarzwaldverein, 1983

ROTT Jean / Euting Julius, in Dictionnaire du monde religieux dans la France contemporaine, tome 2, L'Alsace, Beauchesne, 1987

Suum cuique ! in Strassburger Post, $\mathrm{n}^{\circ}$ 616, 10 juin 1905

Zum Tode Julius Eutings, in Zentralblatt für Bibliothekswesen. XXX. Jahrgang, 3. Heft, März 1913 (Leipzig : Harrassowitz, 1913) 